STRUCTURAL BIOLOGY COMMUNICATIONS

ISSN 2053-230X

Received 14 November 2017

Accepted 30 December 2017

Edited by A. Nakagawa, Osaka University, Japan

Keywords: photosynthesis; 2-Cys peroxiredoxin; oligomeric state; peroxiredoxin 1; atomic force microscopy; Chlamydomonas reinhardtii.

Supporting information: this article has supporting information at journals.iucr.org/f

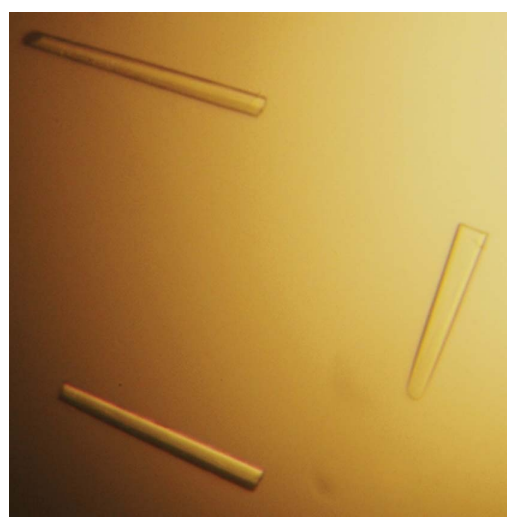

OPEN $\odot$ ACCESS

\section{X-ray crystallographic and high-speed AFM studies of peroxiredoxin 1 from Chlamydomonas reinhardtii}

\author{
Ratana Charoenwattanasatien, ${ }^{\mathrm{a}, \mathrm{b}}$ Hideaki Tanaka, ${ }^{\mathrm{a}}$ Karen Zinzius, ${ }^{\mathrm{c}}$ Ana K. \\ Hochmal, ${ }^{\mathrm{c}}$ Risa Mutoh, ${ }^{\mathrm{d}}$ Daisuke Yamamoto, ${ }^{\mathrm{d}}$ Michael Hippler ${ }^{\mathrm{c}}$ and Genji \\ Kurisu $^{\mathrm{a}, \mathrm{b}}$ *
}

\begin{abstract}
${ }^{\mathbf{a}}$ Institute for Protein Research, Osaka University, Suita, Osaka 565-0871, Japan, ${ }^{\mathbf{b}}$ Graduate School of Science, Osaka University, Toyonaka, Osaka 560-0043, Japan, ' Institute of Plant Biology and Biotechnology, University of Münster, 48143 Münster, Germany, and ' Faculty of Science, Fukuoka University, Nanakuma, Jyonan-ku, Fukuoka 814-0180, Japan. *Correspondence e-mail: gkurisu@protein.osaka-u.ac.jp
\end{abstract}

Peroxiredoxins (PRXs) are a group of antioxidant enzymes that are found in all organisms, including plants and green algae. The 2-Cys PRX from Chlamydomonas reinhardtii ( $\mathrm{Cr} \mathrm{PRX} 1)$ is a chloroplast-localized protein that is critical for clearing reactive oxygen species in chloroplasts. $C r$ PRX1 is reduced by thioredoxins or calredoxin ( $\mathrm{CrCRX})$, a recently identified calcium-dependent redox protein. The molecular interaction between PRXs and thioredoxin/ $\mathrm{CrCRX}$ is functionally important, but discussion has been limited owing to a lack of structural information on $\mathrm{Cr} \mathrm{PRX} 1$, especially regarding its oligomeric state. In this study, high-speed atomic force microscopy (HS-AFM) images of CrPRX1 and an X-ray crystallographic analysis have enabled examination of the oligomeric state of $\mathrm{CrPRX}$. Diffraction data from a crystal of the Cys174Ser mutant of $C r$ PRX1 indicate the existence of noncrystallographic fivefold symmetry. HS-AFM images of $C r P R X 1$ further show that $C r P R X 1$ particles form rings with pentagonal rotational symmetry. On the basis of these findings, the oligomeric state of $\mathrm{CrPRX} 1$ is discussed and it is concluded that this PRX exists in a ring-shaped decameric form comprising a pentamer of dimers.

\section{Introduction}

Photosynthesis is a light-dependent chemical process that converts light energy to chemical energy. Light energy is absorbed by chlorophylls or carotenoids in antenna proteins, and is then transferred to photosystem complexes, either photosystem II or I, which perform charge separation at the core of the complex. In nature, the intensity of light perceived by organisms can fluctuate extensively and sometimes exceeds the photosynthetic capacity of the organism. High-intensity light may lead to the production of reactive oxygen species (ROS) such as singlet oxygen $\left({ }^{1} \mathrm{O}_{2}\right)$, superoxide $\left(\mathrm{O}_{2}^{-\bullet}\right)$, hydroxyl radicals $\left(\mathrm{HO}^{\circ}\right)$ or hydrogen peroxide $\left(\mathrm{H}_{2} \mathrm{O}_{2}\right)$. These ROS are harmful to photosystems and other chloroplast proteins, and may induce photoinhibition (Takahashi \& Badger, 2011). Plants and other organisms have developed several mechanisms to avoid light-induced photoinhibition, such as ROS-scavenging systems (Asada, 2006), phototaxis in green alga (Erickson et al., 2015) and nonphotochemical quenching, which converts the excess light into thermal energy that can be dissipated.

Peroxiredoxins (PRXs) are a group of antioxidant enzymes that are present in all organisms, including plants. In the green alga Chlamydomonas reinhardtii, PRXs are classified into four types based on the number and position of cysteine residues: 
2-Cys PRX, 1-Cys PRX, PRX-Q and type II PRX (Dayer et al., 2008). The 2-Cys PRX from C. reinhardtii (CrPRX1) is a chloroplast-localized protein that is important for detoxifying ROS in the chloroplast. $\mathrm{Cr}$ PRX1 can be activated by various types of thioredoxins (TRX; Sevilla et al., 2015) and its expression is regulated by light and oxygen concentration (Goyer et al., 2002).

In general, the oligomeric state of 2-Cys PRX is a dimer or a decamer, depending on the redox state, protein concentration, $\mathrm{pH}$ or its physiological function (Barranco-Medina et al., 2009). In the crystal structure, the human homologues of 2-Cys PRX, peroxiredoxin 3 (PRX3; PDB entry 5jcg) and peroxiredoxin 4 (PRX4; PDB entry 3tkp), form decamers (Yewdall et al., 2016; Wang et al., 2012). The cryo-TEM structure of PRX3 shows that decameric ring structures are stacked into a long helical filament, which might function as a self-associating chaperone. The crystal structure of 2-Cys PRX from the bacterium Salmonella typhimurium shows that the oxidized enzyme is in the decameric state, and its signalling activity is regulated by a redox-sensitive state change from a dimer to a decamer (Wood et al., 2002). Regarding plants, the crystal structure of PRX (type II) from Populus trichocapa has been reported (Echalier et al., 2005). This plant PRX forms a dimer in both the crystal (PDB entry 1tp9) and in solution, as confirmed by X-ray crystallography and NMR spectroscopy, respectively. Thus, the oligomerization of PRXs is functionally important, but is very difficult to predict based only on aminoacid sequence information.

Recently, we found that $C r$ PRX1 is activated not only by TRXs but also by calredoxin ( $\mathrm{Cr}$ CRX), a novel chloroplastlocalized calcium-dependent protein consisting of calmodulin and thioredoxin domains (Hochmal et al., 2016). A possible mode of interaction between $\mathrm{Cr}$ PRX1 and $\mathrm{CrCRX}$ was proposed on the basis of the crystal structure of $\mathrm{CrCRX}$ (PDB entry 5e37), but the preliminary discussion was limited owing to a lack of structural information on $C r P R X 1$, especially on its oligomeric state. Because $C$. reinhardtii is a model organism in photosynthesis research, structural information on $\mathrm{CrPRX} 1$ would be useful for further analysis of photoacclimation in plant physiology or plant biology, as well as photosynthesis research, and is particularly important for the understanding of $\mathrm{Ca}^{2+}$-dependent electron transfer between $\mathrm{CrPRX} 1$ and $\mathrm{Cr} \mathrm{CRX}$. The aim of the present study was therefore to analyze a single-particle image of recombinant $\mathrm{Cr}$ PRX1 using highspeed atomic force microscopy (HS-AFM) and to crystallize CrPRX1 for further structural analysis. Based on the lowresolution HS-AFM image of $\mathrm{CrPRX} 1$ and the crystallographic analysis described here, we discuss the oligomeric state of $C r$ PRX1.

\section{Materials and methods}

2.1. Cloning, protein expression and purification, and biochemical estimation of molecular weight

The plasmid for the expression of recombinant C. reinhardtii peroxiredoxin 1 ( $\mathrm{Cr} \mathrm{PRX} 1)$ was constructed as described previously (Hochmal et al., 2016) and summarized in Table 1. The pET-22b CrPRX1 plasmid was transformed into Escherichia coli strain BL21(DE3). A single Cys174Ser (C2S) mutant of $C r$ PRX1, which is a mutant that mimics the reduced active-site cysteine residues, was engineered in the pET-22b CrPRX1 plasmid by an In-Fusion Cloning kit (Takara Bio USA, Mountain View, California, USA) using the primers $5^{\prime}$ GAGGTCAGCCCCGCCGGCTGGAAG-3' and $5^{\prime}$-GGC GGGGCTGACCTCATCGGGGTT-3'. Wild-type and C2S

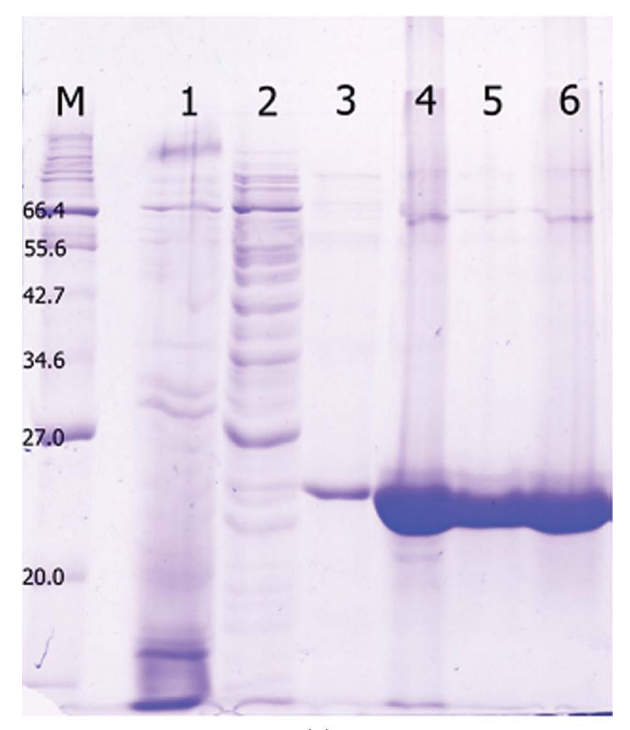

(a)

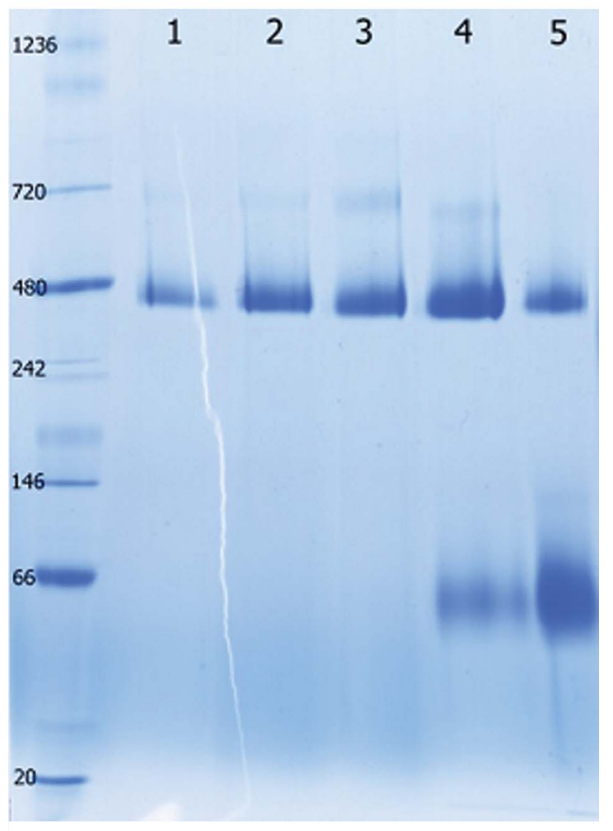

(b)

Figure 1

SDS-PAGE and native PAGE analysis of purified wild-type $C r$ PRX1 protein and its crystals. (a) SDS-PAGE showing the purification of CrPRX1 by Ni-NTA and gel-filtration chromatography. Lane 1, flowthrough from the nickel column. Lanes 2 and 3, fractions from the $20 \mathrm{~m} M$ imidazole wash. Lane 4, elution of CrPRX1 with $250 \mathrm{~m} M$ imidazole. Lanes 5 and 6, fractions from Superdex 200. (b) Native PAGE of $\mathrm{CrPRX} 1$ from dissolved crystals and solution samples. Lanes 1-3, CrPRX1 crystals. Lanes 4 and 5, CrPRX1 from stock protein solution. 
mutant cells were grown in LB medium with $100 \mu \mathrm{g} \mathrm{m}^{-1}$ ampicillin and incubated at $310 \mathrm{~K}$ with shaking until the $\mathrm{OD}_{600}$ reached 0.5-0.6. Protein expression was induced with $0.5 \mathrm{mM}$ isopropyl $\beta$-D-1-thiogalactopyranoside, and the cells were further incubated at $310 \mathrm{~K}$ for $4-6 \mathrm{~h}$. The cells were harvested by centrifugation at $8000 \mathrm{~g}$ for $10 \mathrm{~min}$ and stored at $193 \mathrm{~K}$. The cell pellets were suspended in lysis buffer consisting of $50 \mathrm{~m} M$ Tris- $\mathrm{HCl} \mathrm{pH}$ 8.0, $150 \mathrm{~m} M \mathrm{NaCl}, 1 \mathrm{~m} M$ phenylmethylsulfonylfluoride and disrupted by sonication. The lysate was clarified by ultracentrifugation at $164430 \mathrm{~g}$ for $30 \mathrm{~min}$ and the supernatant was applied onto Ni-NTA resin (Qiagen, Hilden, Germany) pre-equilibrated with $50 \mathrm{~m} M$ Tris- $\mathrm{HCl}$ pH 8.0, $150 \mathrm{~m} M \mathrm{NaCl}$. The Ni-NTA resin was washed and eluted with 20 and $250 \mathrm{~m} M$ imidazole in equilibration buffer. The CrPRX1 protein was further purified using gel filtration on a Superdex 200 HR 16/60 column (GE Healthcare). The column was equilibrated with $20 \mathrm{~m} M$ HEPES pH 7.5, $150 \mathrm{~m} M \mathrm{NaCl}$. Purified protein fractions were concentrated by centrifugation using Amicon filter units with a membrane nominal molecularweight limit of $10 \mathrm{kDa}$ (Merck Millipore, Cork, Ireland) for crystallization. Purified protein fractions were concentrated and kept at $193 \mathrm{~K}$ for crystallization (Fig. 1a).

The molecular weight of wild-type $C r$ PRX1 was first estimated using a gel-filtration column with several protein markers. To verify the estimated molecular weight of $\mathrm{Cr}$ PRX1 both in solution and from dissolved crystal samples, $20 \mu \mathrm{g}$ protein sample or several crystals of $\mathrm{Cr}$ PRX1 were loaded onto gels for SDS-PAGE and native PAGE (NativePAGE Novex 4-16\% Bis-Tris Gels) analysis (Fig. 1). Macromoleculeproduction information is summarized in Table 1.

\subsection{Crystallization}

Crystallization conditions for $\mathrm{Cr}$ PRX1 were screened by the hanging-drop vapour-diffusion method using Crystal Screen, Crystal Screen 2, PEG Rx 1, PEG Rx 2 (Hampton Research, Aliso Viejo, California, USA) and Wizard I, II, III and IV (Rigaku, Bainbridge Island, Washington, USA) at 277 and
Table 1

Macromolecule-production information.

\begin{tabular}{|c|c|}
\hline Source organism & C. reinhardtii \\
\hline DNA source & RNA from $C$. reinhardtii \\
\hline Forward primer & $\begin{array}{l}5^{\prime}-\text { GGTATTCCATATGGCTTCCCACGCCGA } \\
\text { G-3' }\end{array}$ \\
\hline Reverse primer & 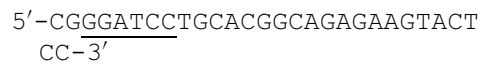 \\
\hline Cloning vector & pET-22b(+) (Novagen) \\
\hline Expression vector & pET-22b(+) (Novagen) \\
\hline Expression host & E. coli strain BL21(DE3) \\
\hline \multirow{8}{*}{$\begin{array}{l}\text { Complete amino-acid sequence } \\
\text { of the construct produced }\end{array}$} & MASHAEKPLVGSVAPDFKAQAVFDQEFQEI \\
\hline & TLSKYRGKYVVLFFYPLDFTFVCPTEIT \\
\hline & AFSDRYKEFKDINTEVLGVSVDSQFTHL \\
\hline & AWIQTDRKEGGLGDLAYPLVADLKKEIS \\
\hline & KAYGVLTEDGISLRGLFI IDKEGVVQHA \\
\hline & TINNLAFGRSVDETKRVLQAIQYVQSNP \\
\hline & DEVCPAGWKPGDKTMKPDPKGSKEYFSA \\
\hline & VQDPNSSSVDKLAAALEHHHHHH \\
\hline
\end{tabular}

293 K. Needle crystals of wild-type $C r$ PRX1 were produced using $0.2 \mathrm{M}$ ammonium acetate, $0.1 \mathrm{M}$ sodium citrate $\mathrm{pH}$ 5.5, $24 \%$ PEG 400 . After optimization including a microseeding technique, crystals with a suitable size for an X-ray experiment were obtained from droplets comprised of $1 \mu \mathrm{l}$ of $30 \mathrm{mg} \mathrm{m}^{-1}$ protein sample and $1 \mu \mathrm{l}$ reservoir solution consisting of $0.05 \mathrm{M}$ ammonium acetate, $0.1 \mathrm{M}$ sodium citrate $\mathrm{pH} 5.5,20 \%$ PEG $400,15 \%$ glycerol (Fig. 2a). Unexpectedly, the crystallization conditions for the C2S mutant of $\mathrm{Cr}$ PRX1 differed markedly from those for the wild type. After optimization of the crystallization conditions, rhombic crystals of the $\mathrm{C} 2 \mathrm{~S}$ mutant were obtained from droplets comprised of $2 \mu \mathrm{l}$ of $15 \mathrm{mg} \mathrm{m}^{-1}$ protein sample and $1 \mu \mathrm{l}$ reservoir solution consisting of $0.1 \mathrm{M}$ HEPES pH 7.6, 0.2 $M$ sodium thiocyanate, 20\% PEG 3350 (Fig. 2b). Crystallization information is summarized in Table 2.

\subsection{X-ray data collection and processing}

Single wild-type $C r$ PRX1 crystals were picked up and directly cooled in liquid nitrogen. Single crystals of the C2S mutant were picked up and first soaked in $20 \%$ glycerol for cryoprotection before being cooled in liquid nitrogen. X-ray

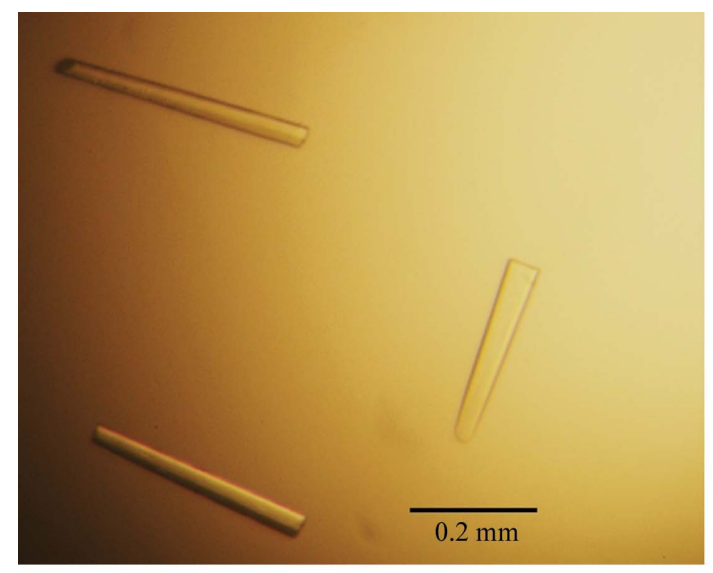

$(a)$

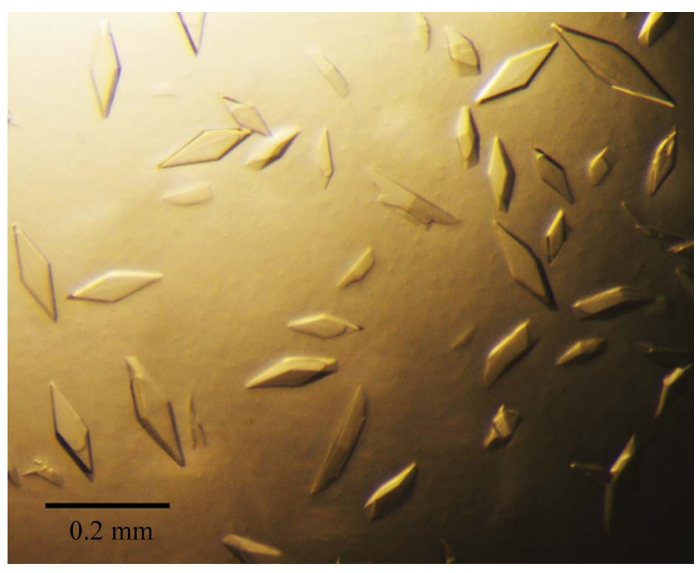

(b)

Figure 2

Photographs of typical crystals of wild-type $C r \mathrm{PRX} 1$ and the C2S mutant. (a) Crystals of wild-type $C r$ PRX1. Crystals were obtained in a droplet consisting of $0.1 \mathrm{M}$ sodium citrate $\mathrm{pH} 7.5,50 \mathrm{mM}$ ammonium acetate, $21 \%$ polyethylene glycol $400,15 \%$ glycerol at $277 \mathrm{~K}$. (b) Crystals of the C2S mutant of $C r$ PRX1. Crystals were obtained in a droplet consisting of $0.1 M$ HEPES pH 7.6, $0.2 M$ sodium thiocyanate, $20 \%$ PEG 3350 at $277 \mathrm{~K}$. 
Table 2

Crystallization.

\begin{tabular}{|c|c|c|}
\hline & Wild type & C2S mutant \\
\hline Method & Hanging-drop vapour diffusion & Hanging-drop vapour diffusion \\
\hline Plate type & Hampton Research 48-well & Hampton Research 48-well \\
\hline Temperature $(\mathrm{K})$ & 277 & 277 \\
\hline Protein concentration $\left(\mathrm{mg} \mathrm{ml}^{-1}\right)$ & 30 & 15 \\
\hline Buffer composition of protein solution & $20 \mathrm{~m} M$ HEPES pH 7.5, $150 \mathrm{~m} M \mathrm{NaCl}$ & $20 \mathrm{~m} M$ HEPES pH 7.5, $150 \mathrm{~m} M \mathrm{NaCl}$ \\
\hline Composition of reservoir solution & $\begin{array}{l}0.05 M \text { ammonium acetate, } 0.1 M \text { sodium citrate } \mathrm{pH} 5.5, \\
20 \% \text { PEG } 400,15 \% \text { glycerol }\end{array}$ & $\begin{array}{l}0.2 M \text { sodium thiocyanate, } 0.1 M \text { HEPES } \mathrm{pH} 7.6 \text {, } \\
20 \% \text { PEG } 3350\end{array}$ \\
\hline Volume and ratio of drop & $1 \mu \mathrm{l}+1 \mu \mathrm{l}$ & $2 \mu \mathrm{l}+1 \mu \mathrm{l}$ \\
\hline Volume of reservoir $(\mu \mathrm{l})$ & 150 & 150 \\
\hline
\end{tabular}

Table 3

Data collection and processing.

Values in parentheses are for the outer shell.

\begin{tabular}{|c|c|c|}
\hline & Wild type & $\mathrm{C} 2 \mathrm{~S}$ mutant \\
\hline Diffraction source & BL44XU, SPring-8 & BL44XU, SPring-8 \\
\hline Wavelength $(\AA)$ & 0.90000 & 0.90000 \\
\hline Temperature (K) & 100 & 100 \\
\hline Detector & Rayonix MX300-HE & Rayonix MX300-HE \\
\hline $\begin{array}{l}\text { Crystal-to-detector distance } \\
(\mathrm{mm})\end{array}$ & 450 & 350 \\
\hline Rotation range per image $\left(^{\circ}\right)$ & 0.5 & 0.3 \\
\hline Total rotation range $\left(^{\circ}\right)$ & 120 & 90 \\
\hline Exposure time per image (s) & 5 & 1 \\
\hline Space group & $P 6_{3}$ & $C 222_{1}$ \\
\hline$a, b, c(\AA)$ & $137.13,137.13,354.87$ & $134.81,418.11,94.32$ \\
\hline$\alpha, \beta, \gamma\left({ }^{\circ}\right)$ & $90,90,120$ & $90,90,90$ \\
\hline Mosaicity $\left(^{\circ}\right)$ & $0.616-0.820$ & 0.197 \\
\hline Resolution range $(\AA)$ & $50-4.80(4.97-4.80)$ & $47.37-2.40(2.45-2.40)$ \\
\hline Total No. of reflections & 131875 & 381061 \\
\hline No. of unique reflections & 18138 & 194153 \\
\hline Completeness (\%) & $99.7(99.9)$ & $96.4(98.8)$ \\
\hline Multiplicity & $7.3(7.1)$ & $1.96(1.96)$ \\
\hline$\langle I / \sigma(I)\rangle$ & $15.3(6.6)$ & $4.65(1.95)$ \\
\hline$R_{\text {merge }}(\%)$ & $13.6(40.7)$ & $9.8(58.4)$ \\
\hline $\begin{array}{l}\text { Overall } B \text { factor from } \\
\text { Wilson plot }\left(\AA^{2}\right)\end{array}$ & 34.797 & 52.6 \\
\hline
\end{tabular}

diffraction images were collected from single crystals of wildtype and C2S mutant CrPRX1 on the BL44XU beamline at SPring-8. Data sets for wild-type CrPRX1 were collected at $100 \mathrm{~K}$ using a CCD-based MX300-HE detector system with an oscillation angle of $0.5^{\circ}$ per frame, a crystal-to-detector distance of $450 \mathrm{~mm}$ and an exposure time of $1 \mathrm{~s}$ per frame. Data sets for C2S mutant CrPRX1 were collected at $100 \mathrm{~K}$ using the same CCD-based detector. The oscillation angle per frame was $0.3^{\circ}$ and the crystal-to-detector distance was $350 \mathrm{~mm}$. The exposure time per frame was $1 \mathrm{~s}$. The diffraction data for the wild type were processed with the $H K L-2000$ software package (Otwinowski \& Minor, 1997) and the data for the C2S mutant were processed with the $X D S$ program package (Kabsch, 2010). Data-collection and processing statistics are summarized in Table 3.

\subsection{High-speed atomic force microscopy (HS-AFM)}

The concentration of $C r \mathrm{PRX} 1$ was $0.1 \mathrm{mg} \mathrm{ml}^{-1}$ in imaging buffer (50 $\mathrm{m} M$ sodium acetate $\mathrm{pH} 5.5,150 \mathrm{mM} \mathrm{NaCl}) .2 \mu \mathrm{l}$ of $100 \mathrm{mM} \mathrm{CoCl}$ was loaded onto freshly cleaved mica substrate for $3 \mathrm{~min}$ at room temperature. After washing the mica surface with imaging buffer, $2 \mu \mathrm{l}$ of $C r$ PRX1 was loaded onto the mica surface and then incubated for $5 \mathrm{~min}$ at room temperature. After washing the mica surface with imaging buffer, HS-AFM imaging was performed in amplitude-modulation mode using a laboratory-built HS-AFM setup. The cantilever used was BL-AC10DS-A2 (Olympus Co., Tokyo, Japan) with a tip fabricated by electron-beam deposition.

\section{Results and discussion}

Recombinant CrPRX1 protein comprising 221 amino acids was successfully overexpressed and purified to electrophoretic homogeneity, showing a molecular weight of $24.7 \mathrm{kDa}$ on SDS-PAGE (Fig. 1a). Native PAGE and gel-filtration chromatographic analysis showed that $C r P R X 1$ exists as an oligomer in solution (Fig. $1 b$ ). The molecular mass of $C r \mathrm{PRX} 1$ from both dissolved crystals and purified solution was estimated to be about $350-480 \mathrm{kDa}$, indicating a clearly greater molecular mass than that of the homodimer, in contrast to the

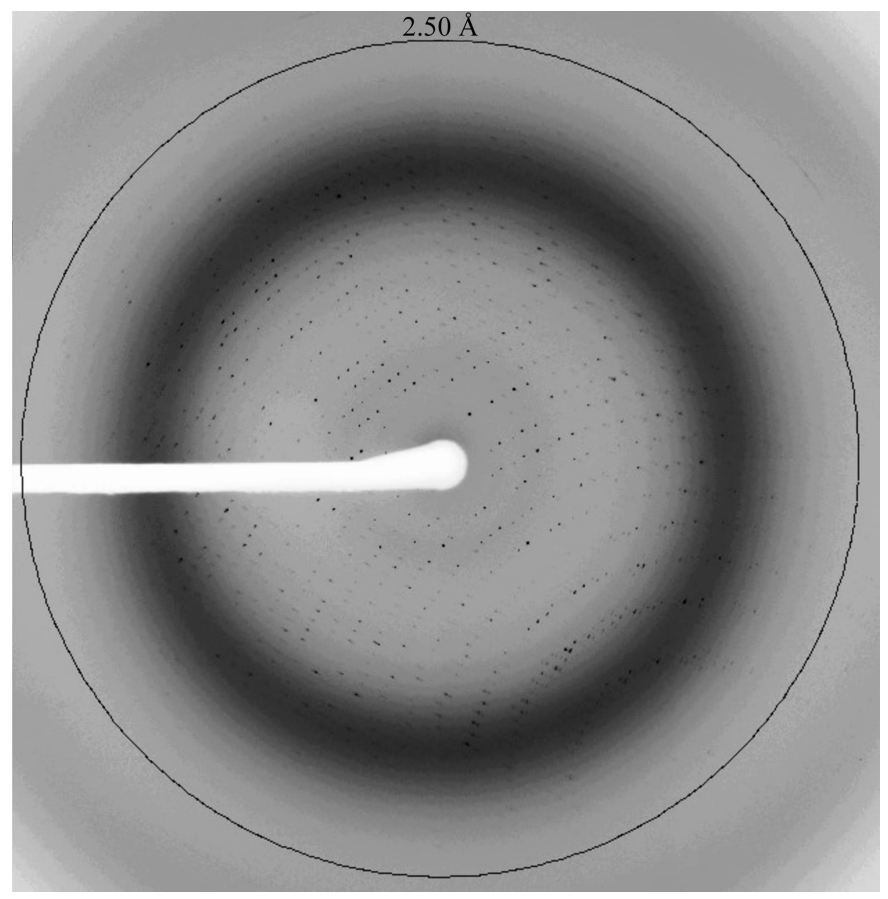

Figure 3

Diffraction image of a $C r P R X 1$ crystal of the $\mathrm{C} 2 \mathrm{~S}$ mutant recorded on BL44XU at SPring-8. 
oligomeric state of type II PRX1 from higher plants (Echalier et al., 2005). However, the solution sample eluted from gelfiltration column chromatography (lanes 4 and 5 in Fig. 1b) also contained a band at about $66 \mathrm{kDa}$ corresponding to the dimeric form. This observation suggests that $C r P R X 1$ can adopt both dimeric and higher oligomeric forms but prefers the oligomeric form, which can be crystallized.

The crystal of wild-type $C r P R X 1$ was found to belong to the hexagonal space group $P 6_{3}$, with unit-cell parameters $a=b=137.12, c=354.87 \AA$, but only diffracted to $4.8 \AA$ resolution (Table 2). In contrast, the crystal of the C2S mutant was found to belong to space group $C 222_{1}$, with unit-cell parameters $a=134.81, b=418.11, c=94.32 \AA$, and diffracted to $2.4 \AA$ resolution, thus being suitable for atomic structure analysis (Fig. 3). This improvement in crystal quality may be owing to the different packing of the $\mathrm{C} 2 \mathrm{~S}$ mutant crystals. For phasing, we carried out molecular replacement using the program Phaser $M R$ in the $C C P 4$ program suite (Collaborative Computational Project, Number 4, 1994; Winn et al., 2011). The best homologous model for the molecularreplacement calculations was thioredoxin peroxidase B from human red blood cells (PDB entry 1qmv; Schröder et al., 2000), with $63 \%$ sequence identity to CrPRX1. Structure determination, including model rebuilding and refinement, is
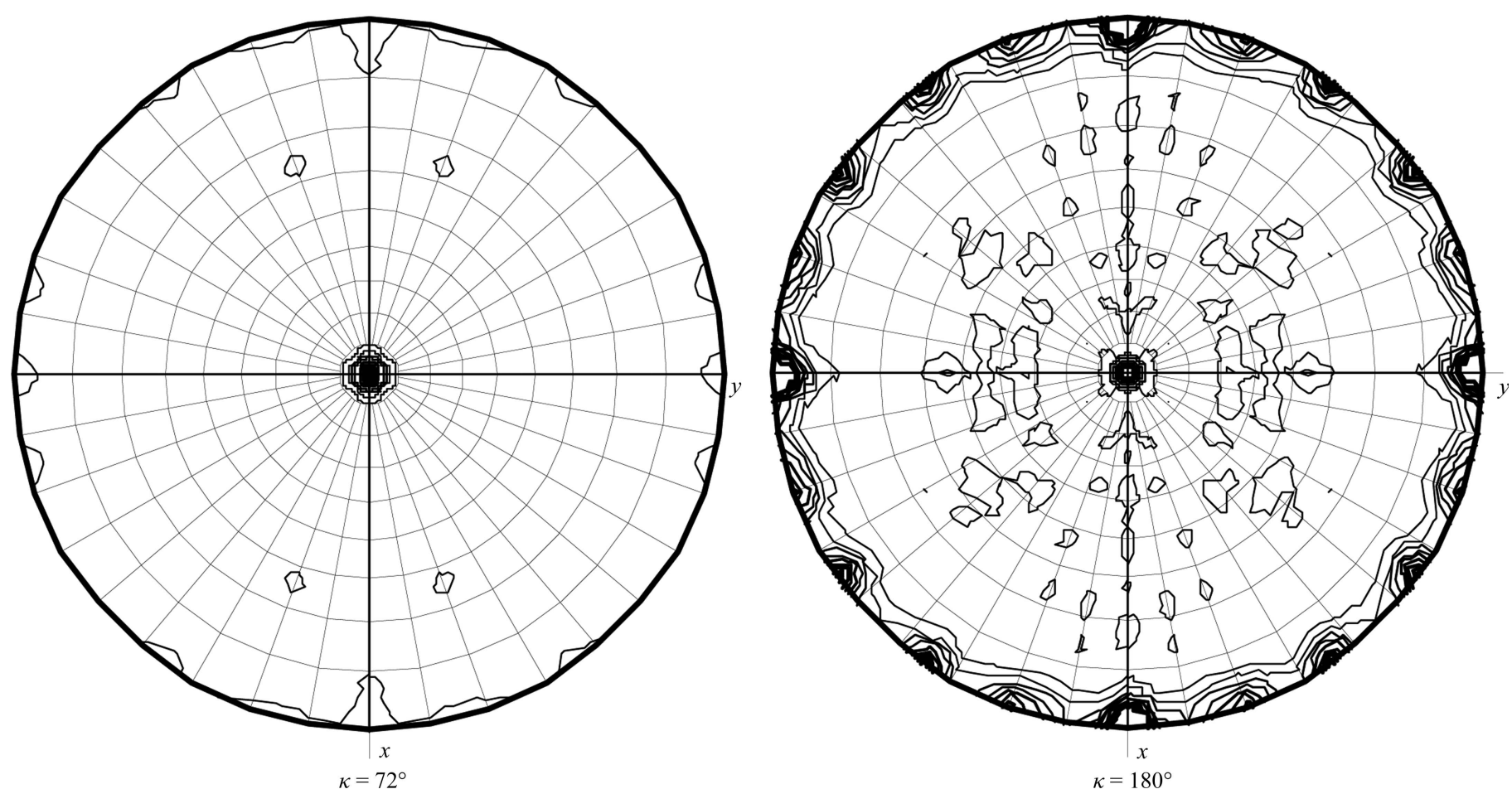

Figure 4

Self-rotation functions of the C2S mutant of $C r P R X 1$ at $\kappa=72^{\circ}$ and $180^{\circ}$. Strong peaks are also found every $72^{\circ}$. The peak heights at the $\kappa=72,144,180$, 216, 288 and $360^{\circ}$ sections are $119700,160200,302200,160200,119700$ and 302 200, respectively.

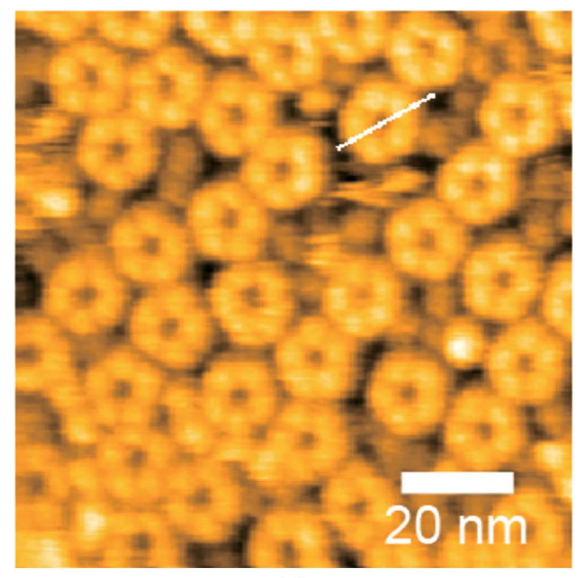

(a)

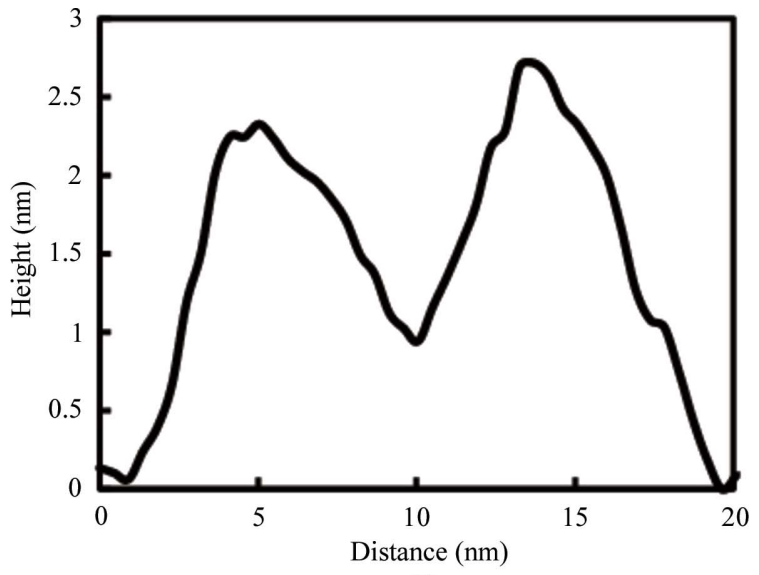

(b)

Figure 5

HS-AFM images of $C r$ PRX1. (a) Topography image. (b) Height profile of a $C r$ PRX1 particle along the white line in $(a)$. The imaging rate was $1 \mathrm{~s}$ per frame. 
now in progress, but we note that the self-rotation functions calculated using the diffraction data from the C2S crystal show an obvious peak in the $\kappa=72^{\circ}$ section corresponding to the existence of noncrystallographic fivefold symmetry (Fig. 4).

Importantly, HS-AFM images revealed that the wild-type CrPRX1 particles formed rings with pentagonal rotational symmetry in solution (Fig. 5). This corresponds well to the fivefold symmetry found in the self-rotation function described above, illustrating that the noncrystallographic fivefold symmetry is intramolecular. The average peak height of AFM images from the mica surface was $2.7 \pm 0.3 \mathrm{~nm}(n=$ 30). The observed outer diameter of the CrPRX1 ring was 16.8 $\pm 0.94 \mathrm{~nm}(n=30)$ on average. Together with the observed fivefold symmetry and the experimentally measured height and diameter of $C r P R X 1$, the possibility of a highly stacked ring structure, as found in the filamentous oligomeric state of PRX3 (Yewdall et al., 2016), is unlikely. Although the only available structure of a plant PRX (that from P. trichocapa) is homodimeric (PDB entry 2pwj; Barranco-Medina et al., 2006), the search model for molecular replacement with the highest sequence identity (PDB entry 1qmv) possessed a decameric ring structure. Considering other examples of 2-Cys PRXs, such as PRX3, PRX4 and PRX5, we conclude that the oligomeric state of $\mathrm{CrPRX} 1$ is a ring-shaped decamer formed by a pentamer of dimers both in the crystal and solution states. Efforts towards structure determination using the molecularreplacement method are currently progressing well, and will provide a clearer insight into the oligomeric state of $C r P R X 1$, as well as being essential for elucidating the functional interaction with TRXs and CrCRX.

\section{Acknowledgements}

We thank the staff of beamline BL44XU at SPring-8, Japan for their help during data collection. X-ray diffraction experiments at SPring-8 were performed under proposal Nos. 2016B6500 and 2017A6500.

\section{Funding information}

Funding for this research was provided by: International Collaborative Research Program, Osaka University (grant to Genji Kurisu, Michael Hippler); CREST-JST, Japan (grant to Genji Kurisu); the German Science Foundation (grant No. DFG HI 739/9-2 to Michael Hippler).

\section{References}

Asada, K. (2006). Plant Physiol. 141, 391-396.

Barranco-Medina, S., Lázaro, J.-J. \& Dietz, K.-J. (2009). FEBS Lett. 583, 1809-1816.

Barranco-Medina, S., López-Jaramillo, F. J., Bernier-Villamor, L., Sevilla, F. \& Lázaro, J.-J. (2006). Acta Cryst. F62, 695-698.

Collaborative Computational Project, Number 4 (1994). Acta Cryst. D50, 760-763.

Dayer, R., Fischer, B. B., Eggen, R. I. \& Lemaire, S. D. (2008). Genetics, 179, 41-57.

Echalier, A., Trivelli, X., Corbier, C., Rouhier, N., Walker, O., Tsan, P., Jacquot, J.-P., Aubry, A., Krimm, I. \& Lancelin, J.-M. (2005). Biochemistry, 44, 1755-1767.

Erickson, E., Wakao, S. \& Niyogi, K. K. (2015). Plant J. 82, 449-465.

Goyer, A., Haslekås, C., Miginiac-Maslow, M., Klein, U., Le Marechal, P., Jacquot, J.-P. \& Decottignies, P. (2002). Eur. J. Biochem. 269, 272-282.

Hochmal, A. K. et al. (2016). Nature Commun. 7, 11847.

Kabsch, W. (2010). Acta Cryst. D66, 125-132.

Otwinowski, Z. \& Minor, W. (1997). Methods Enzymol. 276, 307-326.

Schröder, E., Littlechild, J. A., Lebedev, A. A., Errington, N., Vagin, A. A. \& Isupov, M. N. (2000). Structure, 8, 605-615.

Sevilla, F., Camejo, D., Ortiz-Espín, A., Calderón, A., Lázaro, J.-J. \& Jiménez, A. (2015). J. Exp. Bot. 66, 2945-2955.

Takahashi, S. \& Badger, M. R. (2011). Trends Plant Sci. 16, 53-60.

Wang, X., Wang, L., Wang, X., Sun, F. \& Wang, C.-C. (2012). Biochem. J. 441, 113-118.

Winn, M. D. et al. (2011). Acta Cryst. D67, 235-242.

Wood, Z. A., Poole, L. B., Hantgan, R. R. \& Karplus, P. A. (2002). Biochemistry, 41, 5493-5504.

Yewdall, N. A., Venugopal, H., Desfosses, A., Abrishami, V., Yosaatmadja, Y., Hampton, M. B., Gerrard, J. A., Goldstone, D. C., Mitra, A. K. \& Radjainia, M. (2016). Structure, 24, 1120-1129. 